\title{
Using Biological Analogies for Engineering Problem Solving and Design
}

\author{
L.H. Shu, Associate Professor \\ Dept. of Mechanical \& Industrial Engineering, University of Toronto \\ shu@mie.utoronto.ca
}

\begin{abstract}
This paper summarizes various aspects of identifying and using biological analogies in engineering design. To avoid the immense as well as potentiallybiased task of creating a database specificallyfor this purpose, the chosen approach searches biological knowledge in natural language format, e.g., books, papers, etc., for instances of functionalkeywordsdescribingthe engineeringproblem. Strategiesdevelopedto facilitatethis search as well how text descriptionsof biologicalphenomena were used in problem solving are summarized. Case studies in designfor remanufacture and microassembly are used to provide an overview of the method.
\end{abstract}

\section{Introduction}

Many elegant solutions to engineering problems have been inspired by biological phenomena. While most work in biomimetic design involve specific cases of design that copy particular biological models, not always describedis how these biologicalmodels were identifiedor selected. Therefore, it is possiblethat an engineer open to using biological models for design may not know how to find relevant biological analogies for a given design.

This paper will summarize our past and ongoing work in developing an approach to identify and use relevant biological analogies for any given engineering problem. First highlighted are ongoing challenges in natural language processing and analogical reasoning, and the work towards overcoming these challenges. Analogies identified using this approach for case studies in design for remanufacture and microassembly are then described to illustrate the process.

\section{Method}

The purpose of this work is to develop a generalized methodology by which analogous biological phenomena can be identified and used for any engineering design problem in an objective and repeatable manner. One possible approach to enable generalized biomimetic design is to build a database of biological phenomena for engineering use (Vincent and Mann, 2002). The approach described here avoids the immenseand likely subjectivetask of cataloguing biological phenomena for engineering. Instead, this method takes advantage of the abundant biological information already available in natural language format by searching it directly for relevant phenomena.

This approachhas been implementedin the form of a computerized search tool that locates in biology texts, instancesof functionalkeywordsdescribingthe engineering design problem.

\section{a. Source of biological information}

The initial source of information selected is Life, the Scienceof Biology(Purveset al., 2001), which is the referencetext for the introductorycourse in biology at the University of Toronto. This text is suitable because it is written at a level that can be easily understoodby those with little backgroundin biology. Also, the text covers a large range of organizational levels, from the molecularand cellular,e.g., DNA, to the ecosystem, such that potential solutions are not limited to a particular organizational level.

Other texts can be substitutedor added as required for the initial search. The more challenging task is the initial identification of relevant phenomena. Further detailson such phenomenacan be easily found using more advanced texts and research papers. 


\section{b. Keywords used for search}

Keywordsused to search for relevanttext segments are verbs that describe the desired effect of possible solutions. Verbs are stronglypreferredover nouns as keywords to initiate searches. Searching for nouns typically leads to pre-conceived solutions while searchingfor verbs that describethe desiredaction is more likely to identify biological forms that may not have occurred to the designer.

Synonyms have been used in the past to increase the number of matches. More recent work has found that 'troponyms' produce superior alternative keywords. Troponyms are verbs that describe specific manners of another verb. For example, 'ambling' is a troponym of 'walking' because it is a particular manner of walking (Chiu and Shu, 2004).

\section{Natural language processing}

Despite the advantages, there are challenges involved with using natural-language knowledge sources to identify biological analogies. A primary difficulty involves the quantity and quality of matches.

Even with a single text used as the source, there can be an unmanageable number of matches to the keywords, including synonyms, troponyms, etc. Identifying words that frequently collocate with soughtkeywordscan be used to summarizedominant biological phenomena associated with keywords. For example, high frequency words were "predator", "prey" and "species" for the keyword "eliminate", thus describing how interactions between prey and predator species lead to one another's elimination (Chiu \& Shu, 2004).

Another challenge arises because engineers and biologists use different lexicons or terminology to describe related phenomena. For example, searching for the keyword "clean" for a problem involving cleaning did not locate many useful matches. Therefore, a domain expert was asked for alternative keywords. The keyword, "defend" was suggested since some organisms clean as a defensive mechanism. However,"defend"is relatedto "clean" neither intuitively, for most engineers, nor lexically, e.g., as synonym. Therefore, work was performed towards developing a bridging mechanism that identifies such non-obvious, but highly relevant, biologicallyconnotativekeywordsin an objectiveand repeatable manner that does not require domain experts(Chiu and Shu, 2005). The methodwas used to find "defend" as well as many other biologically connotative keywords for the "clean" example. This bridging method also identified the following biologically connotative keywords: "survive" for an example involving encapsulation, and "break" or "breakdown" for releasing microobjects.

\section{Analogical reasoning}

Anotherchallengein using biologicalanalogiesfor design involves the human process of extracting relevantstrategiesfrom the biologicalphenomenaand applying these strategies to design problems.

Observationsf peopleperformingthese processes revealed four types of similarities between the biological phenomena presented and the concepts developed using them as stimuli. These four similarity types are literal implementation, biological transfer, analogy and anomaly, and are detailed by Mak and Shu (2004a).

Some anomalous concepts result from misunderstanding of the biological phenomenon. Others result from fixating on certain words in the description that are not representative of the overall strategy (Mak and Shu, 2004b). Continuing work seeks to reduce this and other types of fixation that were observed in people attempting to develop analogous concepts from descriptions of biological phenomena.

\section{Examples}

Several analogies for case studies in design for remanufacture(Vakiliand Shu, 2001, Hacco and Shu, 2002) and microassembly (Shu et al., 2003, 2006) were located at various levels of biological organizationby searching for keywords and synonyms related to the engineering problem. These case studies will be briefly described below.

\section{a. Design for remanufacture}

Remanufacture is a process applied to products at their end of life that seeks to reuse product components. Advantages over recycling for scrap materialincludeconservationof resourcesrequiredto melt and reform components. One design guideline identified to facilitate remanufacture is that product features that are prone to failure should be made separable (Shu and Flowers, 1999). In this way, the failed featurescan be replaced, enabling the reuse of a componentwithoutlabor-and capital-intensiveepair operations. However, making failure-prone parts separate increases part count and assembly cost. 
Biological analogies were sought to address this apparent contradiction between ease of assembly and ease of remanufacture.

Searching for the keyword 'remanufacture', not surprisingly, resulted in no matches in the biology text. In this case, alternative keywords such as synonyms are required to find any matches. Other keywords used include 'repair' and 'correct.'

Matches identified using 'repair' involve DNA repair mechanisms, including DNA proofreading during replication, mismatch repair and excision repair. Excision repair was found to be the most analogous to repairs performed during remanufacture.

While the description of excision repair by Purves et al. (2001) confirms relevance of the phenomenonto remanufacture, further research using a more advanced text, Friedberg et al. (1995), was required to determine details that could be used as stimuli for design.

\section{i. Extracting strategy from biological analogy}

The search for the word 'repair' also led to a match in Purveset al. (2001) that providesan analogyat the organism level:

The defense systems of plants and animals differ. Animals generally repair tissues that have been infected. Plants, on the other hand, do not make repairs. Instead, they seal off and sacrifice the damaged tissue so that the rest of the plant does not become infected. This approach works because most plants, unlike most animals, can replace damaged parts by growing new stems, leaves, and roots.

The strategy extracted from the above is based on the ability of plants to add new parts to replace damaged ones, rather than expending resources in repairing damaged parts or sacrificing the entire organism.
Applying this strategy to products involves planning for parts that can be used to replacea brokenfeature, without repairing the broken feature or replacing the entire part that contained the feature.

Anotherphenomenonidentifiedusing the keyword 'correct' involves fainting. The text regarding fainting from Purves et al. (2001) follows:

\begin{abstract}
Blood must be returnedfrom the veins to the heart so that circulation can continue. If the veins are above the level of the heart, gravity helps blood flow, but below the level of the heart, blood must be moved against the pull of gravity. If too much blood remains in the veins, then too little blood returns to the heart, and thus too little blood is pumped to the brain; a person may faint as a result. Fainting is self-correcting: A fainting person falls, thereby moving out of the positionin which gravitycaused blood to accumulate in the lower body.
\end{abstract}

The strategyderivedfrom the above excerptis that fainting is a form of defensive failure that prevents more serious failure.

\section{ii. Applying strategy to engineering system}

Snap fits embody a form of fastening often preferredfor assembly and recyclingpurposes, but are problematicfor remanufacturewhen they fail. Figure 1 compares failed and unbroken snap fits on tonercartridges undergoing remanufacture.

Applyingthe faintingstrategyto the design of snap fits, predeterminedbreakpointsincorporatedinto snap fit configurations as shown in Figure 2a may cause earlier failure than with standard snap fit configurations, but the part containing the snap fit feature can be more easily reused. Applying the ability of plants to sacrifice and replace parts, a possibleplannedreplacemenpart is shown in Figure $2 b$ that can be used once the sacrificial feature in Figure 2a fails.

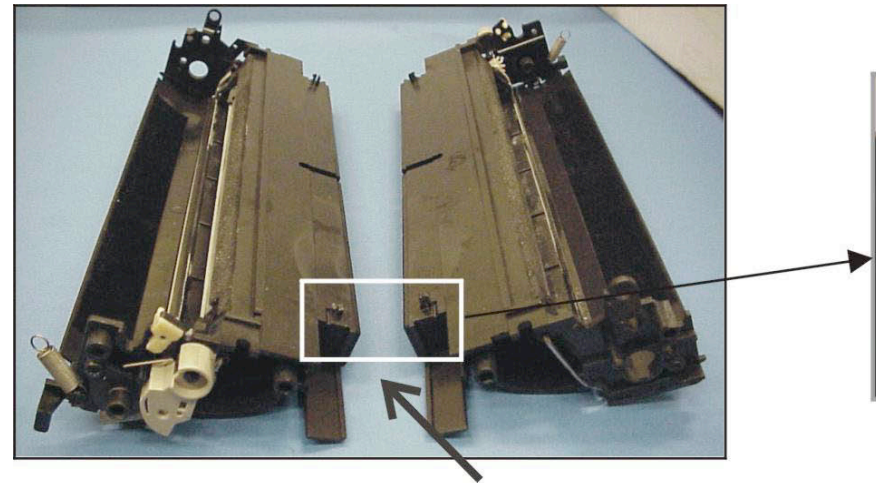

Enlarged Area

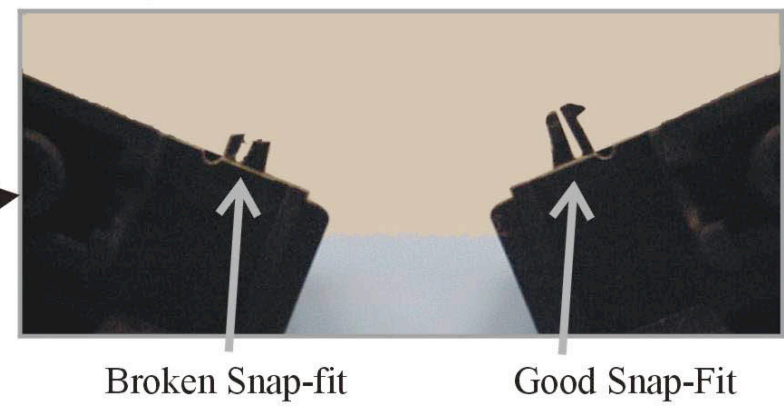

Viewpoint

Figure 1. Damaged snap-fit features (Williams, 2001) 


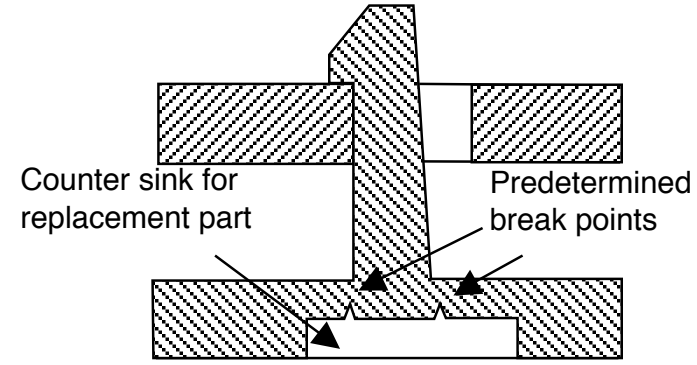

a. Snap fit redesigned with counter sink and break points

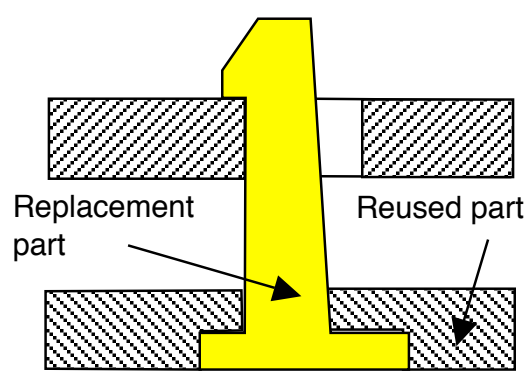

b. Redesigned snap fit after failure and refurbishment

Figure 2. Redesigned snap fit to facilitate repair (Hacco and Shu, 2002)

\section{b. Microassembly}

Collaboration with researchers with expertise in micro- and nanoengineering at the Technical University of Denmark led to the following case studies in microassembly (Shu et al., 2003, 2006).

As the size of products and components become smaller, the manufacture and assembly of such components present challenges beyond those associated with their larger counterparts. For example, in the assembly of microobjects, additional challengesincludethe need for increasedpositional accuracy, the mechanics of interaction between objects, and the loss of direct hand-eye coordination.

Grippers used in microassemby can be roughly categorized as contact and non-contact. Contact grippers make use of principles including mechanicalcontact, vacuum, adhesion, electrostatics and ultrasound. Non-contact grippers make use of principles including optical traps, magnetic and electrical fields. Many of the aforementioned gripping techniques are reported to lack the ability to center objects. Miniaturizedmechanicalgrippers can center objects but present problems including design complexity and not being suitable for clean rooms (Bütefisch and Büttgenbach, 2001).

The goal of the biomimetic search in this example was to identify biologicalphenomenarelevant to the centering of objects within a gripper during microassembly. Relevant phenomena located in Purves et al. (2001), includemicrotubuleorganizing centers, photosystems, and retinal ganglion cells. Details on the use of these stimuli to produce concepts are presented by Shu et al. (2003).

\section{i. Overcoming sticking in microassembly}

Another problem unique to microassembly is that size effects, which occur when part dimensions are scaled down, complicate the handling and assembly of micromechanical parts. A common complicationinvolves sticking between the gripping device and the micropart, which hinders the automation of picking and releasing operations.

Shu et al. (2006) present the identification and use of biologicalanalogiesto solve the problemof sticking during microassembly. Selected release techniques based on DNA transcription and the abscission process in plants inspired concepts of new automated handling devices for microobjects.

The first phenomenonidentifiedis the basis for how proteins are selectively synthesized to defend against infections. This phenomenon involves the interaction between various proteins required to initiate DNA transcription, and led to a concept where features with different geometries would be used to maximizeor minimizesurfacecontactwith the microobjecas needed. However this complex interaction mapped into a relatively complex solution compared to one that can be developed based on the abscission principle described below.

\section{ii. Abscission}

The phenomenon of abscission is the process by which leaves, petals, and fruits separate from a plant. Plants direct growth in different parts such as roots and shoots by strategically releasing a hormone called auxin. When leaves are damaged through infection for example, or are no longer needed, as in the winter season, the leaves stop producingauxin, allowingthe furtherexpressionof abscisic acid and ethylene, which advance abscission. As a result, specific parts of the stalks of the leaves break down and become completely detached from the plant. The base of some leaves contains a special layer of cells called the abscission zone (see Figure 3). In the absenceof auxin, these cells swell and form a cork-likematerial. This cuts off the flow of nutrientsto the leaf and forms a seal 
between the leaf and the plant and protects the plant once the leaf separates (Purves et al., 2001).

The abscission principle can be applied abstractly to microassembly as shown in Figure 4. To overcome difficulties associated with the object adhering to the handling device, the object is releasedtogetherwith a part of the tool designated as sacrificial. The sacrificial part can be of significant mass to take advantage of gravity. In this way the object can be easily released. The sacrificial part of the tool can then either remain with the microobject or be subsequently removed.

For the specificapplicationof insertinga $0.6 \mathrm{~mm}$ metallic microscrew into a plastic counterpart, the abscission zone is physically implemented as a polypropylene rod of $4 \mathrm{~mm}$ diameter that is easily gripped and positioned by a small industrial robot with six degrees of freedom and a specified repeatability of $\pm 0.02 \mathrm{~mm}$ (see Figure 5).

The tip of the polypropylenerod is locally melted by heating, and then pressed over the head of the screw. The contact with the screw results in solidificationof the polypropylene, and a solid bond between the rod and the screw is formed. The robot can now manipulate the screw into the plastic counterpart. Once the screw is tightened into its final position, the resulting increased torque will break the bond between screw and rod.

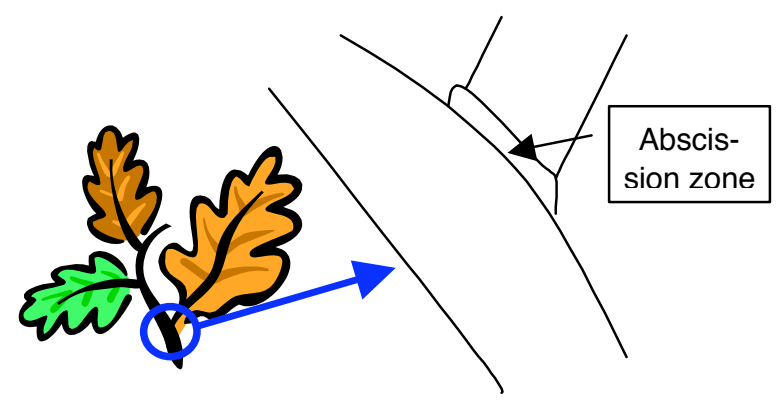

Figure 3. Abscission Zone

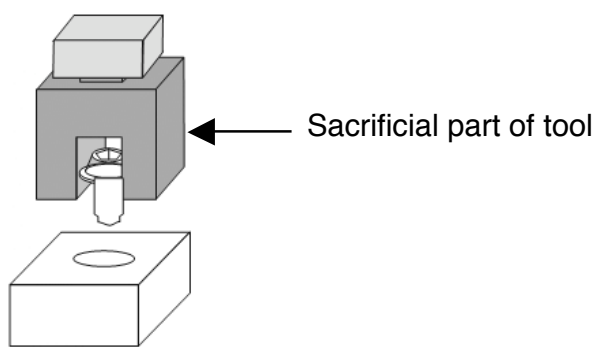

Figure 4. Abscission applied to microassembly

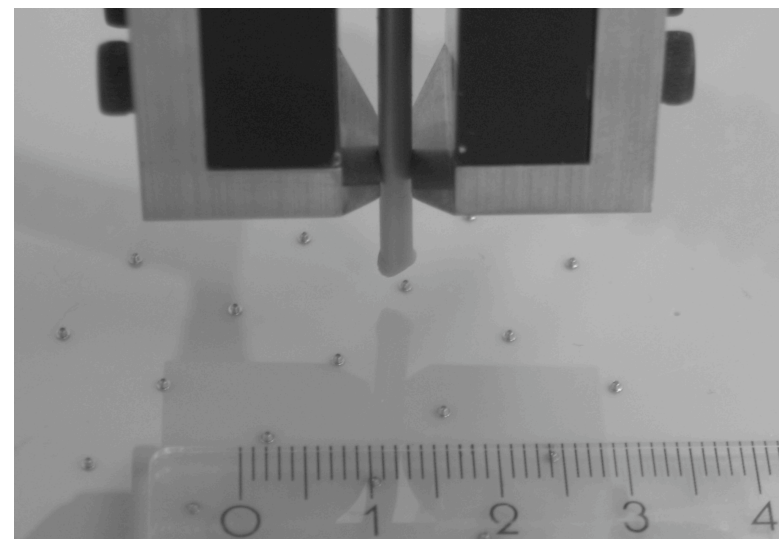

Figure 5. Gripper with polypropylene rod over mounted screws

Other concepts considered for implementation of the abscissionprincipleincluded the use of ice or other intermediatematerial that could be chemically dissolved. This however would introduce possible contaminants that are clearly undesirable. The abscissionprincipleis more broadly interpretedas a physically weaker zone between the tree (gripper) and the leaf (screw). Gravitational, torsional or other forces can be used to break this zone to separate the gripper and screw. In addition to relative "weakness", another factor that led to the choice of a polymer as the intermediate-zone materialis the abilityof polymersto form the very small geometric features that mate with and thus handle the microscrew.

The added challenge of physical implementation of the biological strategy in a solution for this case study highlighted the following. Previous work (Shu et al., 2003) conjectured that biological analogies at a scale comparable to the micro assembly scale could be more directly implemented with less need for abstraction. However, the complex and tightly controlled biological processes identified at the micro scale, only one of which was described for this case study, are difficult to emulate. The far simpler strategy of using an intermediate zone that could be broken down, thermally, chemically or mechanically, was more easily implemented, although the concept of an abscission zone was abstracted into the form of polypropylene rods as opposed to being literally implemented.

This case study suggests that further work should focus on the process of abstracting biological principles and entities to implement as practical engineering solutions, as it may not be possible or desirable to completely emulate analogous biological processes. 


\section{Summary}

This paper summarizedour past and current work in developing a methodology to identify and use relevant biological analogies for any given engineeringproblemin a systematiomanner. First introduced were ongoing challenges and work in languageanalysisand analogicalreasoningin using biological phenomena to develop engineering solutions. Analogies identified using the chosen approach for case studies in design for remanufacture and microassembly were then described to illustrate the process.

\section{Acknowledgments}

Gratefullyacknowledgeds the financialsupport of Natural Sciences and Engineering Research Council of Canada (NSERC).

\section{References}

Bütefisch,S., Büttgenbach,S., 2001, New DifferentialType SMA Actuator for a Miniature Silicon Gripper, Proc. of SPIE, Vol. 4235:102-107.

Chiu, I., Shu, L., 2004, Natural Language Analysis for Biomimetic Design, Proc. of 2004 ASME Design Engineering Technical Conferences and Computers and Information in Engineering Conference, Salt Lake City, Utah, Sept. 28-Oct. 2, 2004, DETC2004/DTM-57250.

Chiu, I., Shu, L., 2005, Bridging Cross-Domain Terminology for Biomimetic Design, Proceedings of 2005 ASME International Design Engineering Technical Conferences and Computers and Information in Engineering Conference, Long Beach, California, USA, September 24-28, 2005. DETC2005/DTM-84908.

Dieter, G.E., 2000, Engineering Design: A Materials and Processing Approach - 3rd edition, McGraw-Hill.

Friedberg,E.C., Walker,G.C., Siede,W., 1995, DNA Repair and Mutagenesis, ASM Press, Washington, D.C.

Hacco, E., Shu, L., 2002, Biomimetic Concept Generation Applied to Design for Remanufacture,Proc. of ASME Design Engineering Technical Conferences and Computers and Information in Engineering Conference, Montreal, Sept. 29-Oct. 2, 2002, DETC2002/DFM-34177.

Mak, T.W., Shu, L.H., 2004a, Abstraction of Biological Analogies for Design, CIRP Annals, Vol. 53/1:117-120.
Mak, T., Shu, L., 2004b, Use of BiologicalPhenomena in Design by Analogy, Proc. of 2004 ASME Design Engineering Technical Conferences and Computers and Information in Engineering Conference, Salt Lake City, Utah, Sept. 28-Oct. 2, 2004, DETC2004/DTM-57303.

Miller, G.A., 1993, Introduction to WordNet: An Online Lexical Database, Five Papers on WordNet, pp. 1-25. ftp://ftp.cogsci.princeton.edu/pub/wordnet/5papers.ps

Purves W.K., Sadava, D., Orians, G.H., Heller, H.C., 2001, Life, The Science of Biology, 6/e, Sinauer Associates, Sunderland, MA.

Shu, L. and Flowers, W., 1999, Application of a Design-for-Remanufacture Framework to the Selection of Product Life-Cycle Fastening and Joining Methods, InternationalJournal of Robotics and Computer Integrated Manufacturing (Special Issue: Remanufacturing), 15/3:179-190.

Shu, L.H., Lenau, T.A., Hansen, H.N., Alting, L., 2003, Biomimetics Applied to Centering in Microassembly, CIRP Annals, 52/1:101-104.

Shu, L.H., 2004, Biomimetic Design for Remanufacture in the Context of Design for Assembly, Proceedings of the I Mech E Part B: Journal of Engineering Manufacture, 218/3:349-352. (Invited Paper)

Shu, L, Hansen, H., Gegeckaite, A., Moon, J., Chan, C., 2006, Case Study in Biomimetic Design: Handling and Assembly of Microparts, ASME 2006 International DesignEngineeringTechnicalConferences\& Computers and Information in Engineering Conference, September 10-13, 2006, Philadelphia, PA, USA, DETC2006-99398

Vakili, V., Shu, L, 2001, Towards BiomimeticConcept Generation, Proc. of 2001 ASME Design Technical Conferences, Design Theory and Methodology Conference, Pittsburgh, PA, Sept. 9-12, 2001, DETC2001/DTM-21715.

Vincent, J., Mann, D., 2002, Systematic Technology Transfer from Biology to Engineering, Philosophical Trans. of The Royal Society: Physical Sciences, 360: 159-173.

Williams, J., 2001, Quantification and Analysis of Remanufacturing Waste Streams for Improving Product Design, Master of Applied Science Thesis, University of Toronto, Department of Mechanical and Industrial Engineering. 